\title{
Surgical intervention for pituitary apoplexy: an analysis of functional outcomes
}

\author{
Martin J. Rutkowski, MD, Sandeep Kunwar, MD, Lewis Blevins, MD, and Manish K. Aghi, MD, PhD \\ California Center for Pituitary Disorders, Department of Neurological Surgery, University of California, San Francisco, California
}

OBJECTIVE Pituitary apoplexy is a clinical syndrome consisting of neurological and endocrine abnormalities secondary to hemorrhage or ischemia of an underlying pituitary adenoma. The authors investigated whether there was a significant difference in neurological, endocrine, and nonneuroendocrine outcomes for patients with pituitary apoplexy, based on the time between symptom onset and surgical intervention.

METHODS The authors retrospectively analyzed the medical records of 32 patients who had presented to their institution with acute pituitary apoplexy and subsequently undergone endonasal transsphenoidal resection in the period from 2003 to 2014. All patients had undergone preoperative MRI demonstrating evidence of apoplexy in the form of intratumoral hemorrhage, ischemia, and necrosis. Neurological deficits, partial or complete endocrinopathy, and nonneuroendocrine abnormalities were analyzed both pre- and postoperatively.

RESULTS Preoperatively, neurological deficits including visual loss and cranial nerve palsies were found in $31(97 \%)$ of the 32 patients, endocrinopathy in the form of partial or panhypopituitarism was seen in 28 patients ( $88 \%)$, and nonneuroendocrine signs and symptoms were seen in 32 patients $(100 \%)$. Thirteen patients $(41 \%)$ underwent surgery within 72 hours of symptom onset ("early"), whereas 19 patients (59\%) underwent surgery more than 72 hours from symptom onset ("delayed"). Early versus delayed resection did not appear to significantly improve visual deficits, total visual loss, resolution of oculomotor palsy, recovery from hypopituitarism, or nonneuroendocrine signs and symptoms such as headache and encephalopathy. Overall, visual improvement was seen in $77 \%$ of patients, complete restoration of normal vision in $38 \%$ of patients, and resolution of preoperative oculomotor palsies in $81 \%$ of patients. Only $6(21 \%)$ of 28 patients showed evidence of partial hormone recovery following preoperative hypopituitarism. An absence of benefit for early surgery held true even when considering time to surgery from symptom onset as a continuous variable.

CONCLUSIONS Neurological deficits such as visual loss and cranial neuropathies show moderate improvement following surgical decompression, as does preoperative hypopituitarism. The timing of surgical intervention relative to the onset of symptoms does not appear to significantly affect the resolution of neurological or endocrinological deficits.

https://thejns.org/doi/abs/10.3171/2017.2.JNS1784

KEY WORDS apoplexy; deficit; endocrine; neurological; pituitary surgery; visual

$\mathrm{P}$ ITUITARY apoplexy is a clinical syndrome consisting of neurological deficits and endocrine abnormalities secondary to hemorrhage and/or ischemia of an underlying pituitary adenoma, resulting in tumor necrosis, edema, and expansion. ${ }^{22,25,26,36}$ Neurological deficits may be directly attributable to increased intrasellar pressure from adenoma expansion with resultant mass effect on adjacent cranial nerves (CNs). The loss of visual acuity and visual field deficits are caused by suprasellar expansion with pressure on the optic nerves or chiasm, while diplopia, ptosis, and partial or complete ophthalmoplegia can result from mass effect on CNs III, IV, and VI. ${ }^{11}$ Endocrinopathy in the form of partial or panhypopituitarism is also commonly encountered, likely from critical glandular compression following intratumoral hemorrhage or from necrosis of intrasellar contents following ischemia. Additionally, patients can present with severe headache, nausea, vomiting, lethargy, obtundation, and even nuchal rigidity mimicking subarachnoid hemorrhage.

With an incidence ranging from $2 \%$ to $20 \%$ among pa-

ABBREVIATIONS CN = cranial nerve; GTR = gross-total resection; $S T R$ = subtotal resection

SUBMITTED January 9, 2017. ACCEPTED February 24, 2017.

INCLUDE WHEN CITING Published online September 15, 2017; DOI: 10.3171/2017.2.JNS1784. 
tients presenting with pituitary adenoma, ${ }^{13}$ apoplexy has classically been considered an acute medical and surgical emergency. In its most extreme form, pituitary apoplexy can be life-threatening due to acute hypocortisolemia from the sudden loss of adrenocorticotropic hormone (ACTH) production, acute or chronic hypothyroidism, and severe hyponatremia or diabetes insipidus. Reversal of acute vision loss or ophthalmoplegia is also a commonly cited reason for emergent resection and decompression of apoplectic tumors. Nevertheless, there remains an unclear association between time to surgical intervention and rates of neurological and endocrine recovery. The relatively limited case series in the literature have also made it difficult to ascertain predisposing factors for pituitary apoplexy, although larger tumor size, anticoagulation, patient age, hypertension, pregnancy, and diabetes mellitus have been implicated. ${ }^{23,26,32,34}$ Further complicating analyses of pituitary apoplexy, some studies have divided patients into those with acute versus subacute apoplexy. Acute apoplexy-the development of sudden symptoms with evidence of mass effect from an associated hemorrhagic or infarcted adenoma-has been distinguished from subclinical apoplexy $9,25,37$ - a minimally symptomatic or asymptomatic patient with evidence of an infarcted or hemorrhagic tumor-but the clinical significance of this distinction remains unclear.

Despite efforts to operate in an urgent fashion, there is often a time delay between the onset of symptoms and transsphenoidal surgery for acute pituitary apoplexy, often because of delayed patient presentation to a health care provider or emergency room or because of delays in patient transfers to centers able to provide comprehensive surgical and medical care for apoplexy. In an effort to determine the correlation between functional outcomes in patients with acute pituitary apoplexy and time from symptomatic onset to surgery, we report our experience in the surgical management of acute pituitary apoplexy.

\section{Methods \\ Study Design}

We performed a retrospective observational study of our series of pituitary apoplexy patients. All patients were enrolled and consented to research participation under approval of our university's institutional review board, known as the Committee on Human Research.

\section{Patient Cohort}

We retrospectively reviewed the records of all patients who had undergone treatment of pituitary adenoma in the period from 2003 to 2014, identifying 32 patients who had presented with acute pituitary apoplexy, all of whom underwent endonasal transsphenoidal resection of a pathologically confirmed necrotic and/or hemorrhagic pituitary adenoma. Patients were followed up until 2014, and data collection and analysis were performed in 2016.

All patients had undergone preoperative MRI or CT demonstrating evidence of apoplexy in the form of intratumoral hemorrhage, ischemia, and necrosis. Patient records were further reviewed to collect demographic data including age, sex, timing of symptom onset, neurological defi- cits, endocrinopathies, nonneuroendocrine symptoms, hyponatremia, treatment course, postoperative hormone replacement, and follow-up duration. Only patients with the onset of clinical symptoms secondary to mass effect from apoplectic adenomas were included in our case series.

\section{Tumor Characteristics}

Data on tumor characteristics such as size, functionality (hormonally hypersecreting versus nonfunctional), pathological staining, invasion, extent of resection, and recurrence rates were collected and analyzed. Functionality was determined on the basis of histological staining in conjunction with laboratory evidence of an abnormal preoperative hormonal elevation. Cavernous sinus invasion was assessed using the Knosp criteria,${ }^{14}$ with Grades 3 and 4 (tumor invasion beyond a line tangential to the lateral margins of the cavernous internal carotid artery, and total internal carotid artery encasement, respectively) designating positive cavernous sinus invasion. Suprasellar invasion was defined as tumor displacement or abutment of the optic chiasm. Recurrence was determined radiologically, defined as either MRI evidence of tumor reappearance following primary gross-total resection (GTR) or as MRI evidence of tumor progression of known residual disease following primary subtotal resection (STR). Time until recurrence was defined as the time from primary resection until the most recent MRI study showing absent disease, stable residual disease, or recurrent disease.

\section{Symptom Classification and Outcome}

Signs and symptoms attributable to pituitary apoplexy were subdivided into 3 groups: 1) neurological, 2) endocrine, and 3) nonneuroendocrine. Recorded neurological signs and symptoms included those referable to local mass effect from apoplectic tumors including dysfunction of CN II (blurry vision, field cuts, blindness), CN III (diplopia, ptosis, mydriasis, ophthalmoplegia), CN IV (diplopia, ophthalmoplegia), and CN VI (diplopia, ophthalmoplegia). Postoperative neurological outcomes were further analyzed by recovery of 1) CN II function (visual changes) and 2) CN III, IV, and VI function (cranial neuropathies). Endocrine signs and symptoms were assessed using preand postoperative laboratory testing to determine rates of hypopituitarism, hyponatremia, diabetes insipidus, adenoma functionality, and the need for partial or complete postoperative hormone replacement. Nonneuroendocrine signs and symptoms were recorded and included preoperative rates of headache, nuchal rigidity, nausea and/or vomiting, and encephalopathy (altered mental status).

\section{Early Versus Delayed Surgical Intervention}

"Early" surgical intervention was defined as resection performed within 72 hours of symptom onset, whereas "delayed" surgical intervention was defined as resection beyond 72 hours of symptom onset. Given that only 2 patients in our cohort underwent resection within 24 hours of symptom onset, the 72-hour time point was chosen to stratify patients into reasonably sized cohorts undergoing surgery in an early versus delayed manner relative to symptom onset. Improvements in neurological and endo- 
crine signs and symptoms were each analyzed by early versus delayed surgical intervention.

\section{Statistical Analysis}

All statistical analysis was performed using SPSS Statistics Developer 23.0 (IBM Corp.) with significance defined as $p<0.05$. Chi-square and Fisher's exact tests were used for categorical variables, the Student t-test for continuous variables, and the log-rank test for recurrence data.

\section{Results \\ Patient Cohort and Presentation}

From 2003 to 2014, 32 patients presented with acute pituitary apoplexy. There were 21 males $(66 \%)$ and $11 \mathrm{fe}-$ males (34\%), ranging in age from 10 to 79 years old (mean age 49 years).

Neurological deficits were found in 31 (97\%) of 32 patients preoperatively: 25 patients $(78 \%)$ suffered blurry vision, 15 patients $(47 \%)$ suffered field cuts and/or subjective visual loss, and 16 patients (50\%) suffered from CN III, IV, and/or VI neuropathy (Table 1). Of those patients with vision loss, $6(40 \%)$ suffered acute vision loss within 24 hours of symptom onset, including 1 case of monocular blindness and 5 cases of binocular field cuts and/or blindness; 9 patients $(60 \%)$ suffered progressive vision loss over days to weeks consisting of blurriness and/or worsening visual acuity, including 3 cases of binocular blindness, 2 cases of monocular blindness, 1 case of a monocular field cut, 1 case of bitemporal hemianopia, and 2 cases of worsened binocular visual acuity.

Endocrinopathy in the form of partial or panhypopituitarism was seen in 28 patients $(88 \%)$ preoperatively (Table 1). More specifically, hypothyroidism was seen in 13 $(43 \%)$ of the 30 patients with available preoperative thyroid levels, and hypocortisolemia was noted in $15(71 \%)$ of the 21 patients with available preoperative cortisol levels. Hyponatremia was found in 15 patients $(47 \%)$ on presentation, including 9 patients with serum sodium values measuring 130-134 mmol/L and 6 patients with values measuring $<130 \mathrm{mmol} / \mathrm{L}$. One patient $(3 \%)$ presented with diabetes insipidus.

Nonneuroendocrine signs and symptoms were also common (Table 1), seen in all 32 patients $(100 \%)$. Headache occurred in 32 patients (100\%), nausea and vomiting in 10 patients $(31 \%)$, encephalopathy in 6 patients $(19 \%)$, and nuchal rigidity in 4 patients $(12 \%)$. Twenty-three patients $(72 \%)$ suffered the acute onset of headaches, while the other 9 patients $(28 \%)$ presented with subacute headaches that progressively worsened. Two patients $(6 \%)$ were intoxicated on methamphetamine on presentation. Initially, 5 patients had workups for subarachnoid hemorrhage prior to the diagnosis of apoplexy, 4 of whom (80\%) showed evidence of increased cerebrospinal fluid leukocytes, erythrocytes, and protein on lumbar puncture.

\section{Neuroimaging Features}

As assessed on preoperative MRI, 22 (73\%) of 30 patients showed evidence of intratumoral intrinsic T1 hyperintensity indicative of hemorrhage and blood products. The presence of intratumoral hemorrhage was not associ-
TABLE 1. Rates of pre- and postoperative deficits among apoplexy patients

\begin{tabular}{ccc}
\hline Signs \& Symptoms & Preop & Postop \\
\hline Neurological & $97 \%$ & \\
\hline Vision loss or blurriness & $97 \%$ & $23 \%$ \\
\hline CN III, IV, \&/or VI neuropathy & $50 \%$ & $24 \%$ \\
\hline Endocrinopathy & $88 \%$ & $69 \%^{*}$ \\
\hline Hyponatremia & $47 \%$ & $\mathrm{NA}$ \\
\hline Non-NE & $100 \%$ & \\
\hline Headache & $100 \%$ & $19 \%$ \\
\hline Nausea/vomiting & $31 \%$ & $0 \%$ \\
\hline Encephalopathy & $19 \%$ & $0 \%$ \\
\hline Nuchal rigidity & $12 \%$ & $0 \%$ \\
\hline
\end{tabular}

$\mathrm{NA}=$ data not available; $\mathrm{NE}=$ neuroendocrine .

* As determined among 18 patients with available hormone follow-up performed at least 6 months postoperatively.

ated with preoperative visual loss $(\mathrm{p}=1.0$, Fisher's exact test), preoperative CN III, IV, or VI neuropathy ( $\mathrm{p}=0.689$, Fisher's exact test), preoperative hypopituitarism $(\mathrm{p}=1.0$, Fisher's exact test), or time (days) from symptom onset to surgery $(\mathrm{p}=0.548, \mathrm{t}$-test $)$.

Furthermore, the presence of hemorrhage did not have an effect on visual improvement $(\mathrm{p}=1.0$, Fisher's exact test), total visual recovery ( $\mathrm{p}=1.0$, Fisher's exact test), improved oculomotor palsies ( $\mathrm{p}=1.0$, Fisher's exact test), endocrine recovery ( $\mathrm{p}=1.0$, Fisher's exact test), and nonneuroendocrine symptoms $(\mathrm{p}=0.647$, Fisher's exact test) including postoperative headaches $(\mathrm{p}=1.0$, Fisher's exact test).

Seventy-one percent of patients showed evidence of sphenoid sinus mucosal inflammation, thickening, and contrast enhancement, features that have been previously associated with apoplectic tumors. ${ }^{16}$

\section{Baseline Demographics and Rates of Early Versus Delayed Treatment}

All patients underwent endonasal transsphenoidal resection of their apoplectic pituitary adenomas. Early surgery, defined as resection within 72 hours of symptom onset, occurred in 13 patients (41\%), whereas delayed surgery, defined as resection more than 72 hours from symptom onset, occurred in 19 patients (59\%), including 3 (9\%) in whom surgery was performed at 2-3 weeks from the time of symptom onset (Table 2). When comparing baseline characteristics between early versus delayed surgery cohorts, there were no significant differences in patient sex $(p=0.283$, Fisher's exact test $)$ or age $(p=0.328$, t-test $)$.

\section{Pathology}

Five tumor samples (16\%) sent for pathology lacked enough viable tumor to establish biochemical diagnosis. Among the 27 successfully tested tumor samples with viable tissue, there were 19 nonfunctional adenomas (70\%), 4 lactotrophs (15\%), 1 somatotroph (4\%), 2 corticotrophs (7\%), and 1 mixed lactotroph/somatotroph (4\%; Table 3). Four patients $(15 \%)$ had clinically hypersecreting adeno- 
TABLE 2. Comparison of baseline characteristics between early and delayed treatment groups as defined by resection earlier or later than 72 hours from the time of symptom onset, respectively

\begin{tabular}{lccc}
\hline \multicolumn{1}{c}{ Parameter } & Early Surgery & Delayed Surgery & $\mathrm{p}$ Value \\
\hline No. of patients & $13(41 \%)$ & $19(59 \%)$ & \\
\hline Sex & $6 \mathrm{~F} / 7 \mathrm{M}$ & $5 \mathrm{~F} / 14 \mathrm{M}$ & 0.283 \\
\hline Mean age in yrs & 45 & 51 & 0.328 \\
\hline Mean tumor size in cm & 3.1 & 2.8 & 0.177 \\
\hline Tumor functionality & $8 \%$ & $16 \%$ & 0.629 \\
\hline Cavernous sinus invasion & $17 \%$ & $40 \%$ & 0.128 \\
\hline Suprasellar invasion & $100 \%$ & $95 \%$ & 1.0 \\
\hline Patients w/ GTR & $100 \%$ & $44 \%$ & $\mathbf{0 . 0 0 3}$ \\
\hline Preop hypopituitarism & $69 \%$ & $100 \%$ & $\mathbf{0 . 0 2}$ \\
\hline
\end{tabular}

Boldface type indicates statistical significance.

* Based on available postoperative scans in 27 of the 32 patients: 11 of 11 patients who underwent early surgery and 7 of 16 patients who underwent delayed surgery.

mas including 3 prolactinomas (11\%) and 1 somatotrophinoma (4\%). Two patients (6\%) were diagnosed with atypical tumors on final pathology, and both were found to be prolactinomas. One patient with a known nonatypical prolactinoma had been medically managed with cabergoline but nonetheless progressed to apoplexy.

\section{Extent of Resection for Early Versus Delayed Treatment}

Postoperative scans were available in 27 of 32 patients, confirming 18 GTRs (67\%) and 9 STRs (33\%). There was a significant difference in the extent of resection in the early versus delayed surgery subgroups (Table 2). Gross-total resection was achieved in $11(100 \%)$ of 11 patients undergoing early surgery versus 7 (44\%) of 16 patients undergoing delayed surgery ( $p=0.003$, Fisher's exact test), despite the absence of significant baseline differences between the early and delayed groups in terms of tumor size $(p=0.177$, $\mathrm{t}$-test), cavernous sinus invasion ( $\mathrm{p}=0.128$, Fisher's exact test), or suprasellar invasion ( $\mathrm{p}=1.0$, Fisher's exact test). Intratumoral hemorrhage on presentation had no effect on rates of resection ( $p=0.382$, Fisher's exact test).

\section{Visual Outcomes for Early Versus Delayed Treatment}

Among patients undergoing early versus delayed resection, there was no significant difference in the baseline rate of preoperative visual dysfunction $(92 \%$ vs $100 \%$, respectively, $\mathrm{p}=0.406$, Fisher's exact test) including more specifically visual loss and field cuts $(69 \%$ vs $32 \%$, respectively, $p=0.070$, Fisher's exact test). Early resection did not appear to significantly improve visual signs and symptoms as compared with delayed resection: visual improvement was seen in $8(67 \%)$ of 12 patients undergoing early surgery versus $16(84 \%)$ of 19 patients undergoing delayed surgery ( $p=0.384$, Fisher's exact test; Table 4). Complete resolution of preoperative decreased visual acuity or field cuts was seen in $4(33 \%)$ of 12 patients undergoing early surgery versus 7 (41\%) of 17 undergoing delayed surgery $(p=0.717$, Fisher's exact test). Overall, $38 \%$ of patients with preoperative decreased visual acuity or field cuts
TABLE 3. Baseline characteristics among 27 patient with apoplectic adenomas*

\begin{tabular}{cc}
\hline \multicolumn{1}{c}{ Variable } & Value \\
\hline Mean tumor size in cm (range) & $2.9(1.8-4.1)$ \\
\hline Macroadenoma & $100 \%$ \\
\hline Cavernous sinus invasion & $34 \%$ \\
\hline Suprasellar invasion & $97 \%$ \\
\hline Nonfunctional adenoma & $70 \%$ \\
\hline Clinically hypersecreting adenoma & $15 \%$ \\
\hline Prolactinoma & $11 \%$ \\
\hline Somatotrophinoma & $4 \%$ \\
\hline Hormone+ pathology & $30 \%$ \\
\hline Prolactin+ & $15 \%$ \\
\hline Growth hormone+ & $4 \%$ \\
\hline ACTH+ & $7 \%$ \\
\hline Prolactin+/growth hormone+ & $4 \%$ \\
\hline
\end{tabular}

ACTH = adrenocorticotropic hormone; + = positive.

* Twenty-seven patients had enough viable tumor to establish a biochemical diagnosis.

achieved complete restoration of their vision, regardless of time from symptom onset to surgery.

There were no significant differences in the baseline rates of preoperative CN III, IV, and VI neuropathies ( $\mathrm{p}=$ 1.0, Fisher's exact test) between the patient cohorts. Neither was early resection associated with improvement in $\mathrm{CN}$ palsies as compared with delayed resection: 5 (83\%) of 6 early surgery patients with preoperative palsies showed $\mathrm{CN}$ improvement versus $8(80 \%)$ of 10 delayed surgery patients ( $\mathrm{p}=1.0$, Fisher's exact test; Table 4).

\section{Endocrine Outcomes for Early Versus Delayed Treatment}

There was a significant difference in the rate of preoperative hypopituitarism between the early and delayed treatment patients (Table 2). While $9(69 \%)$ of 13 patients undergoing early surgery suffered preoperative hypopituitarism, all 19 patents (100\%) undergoing delayed surgery suffered preoperative hypopituitarism ( $\mathrm{p}=0.02$, Fisher's exact test). Nevertheless, among patients with preoperative hypopituitarism, there was no significant difference in the rate of hormone recovery between those undergoing early versus delayed surgery ( $30 \%$ vs $20 \%, \mathrm{p}=0.653$ Fisher's exact test; Table 4) or in the time until hormone recovery (80 vs 72 months, $p=0.784$, log-rank). Recovery tended to happen relatively early posttreatment: 5 of 6 patients recovered function within 7 months, whereas 1 patient showed evidence of recovery at 16 months. Endocrine follow-up ranged from 1 to 121 months postoperatively, with an average of 7 years.

\section{Outcomes for Nonvisual and Nonendocrine Symptoms for Early Versus Delayed Treatment}

There were no significant differences in preoperative nonneuroendocrine symptoms, including headache $(\mathrm{p}=$ 1.0, Fisher's exact test), encephalopathy ( $\mathrm{p}=0.194$, Fisher's exact test), nuchal rigidity ( $\mathrm{p}=1.0$, Fisher's exact test), or 
TABLE 4. Comparison of neurological, endocrine, and nonneuroendocrine deficits between early and delayed surgery groups

\begin{tabular}{lccc}
\hline \multicolumn{1}{c}{ Outcome } & $\begin{array}{c}\text { Early } \\
\text { Surgery }\end{array}$ & $\begin{array}{c}\text { Delayed } \\
\text { Surgery }\end{array}$ & $\begin{array}{c}\mathrm{p} \\
\text { Value }\end{array}$ \\
\hline Neurological deficit & & & \\
\hline$\quad$ Improved vision & $67 \%$ & $84 \%$ & 0.384 \\
\hline$\quad$ Total resolution of visual deficits & $33 \%$ & $41 \%$ & 0.717 \\
\hline$\quad$ Improved cranial neuropathies & $83 \%$ & $80 \%$ & 1.0 \\
\hline Endocrinopathy & & & \\
\hline$\quad$ Improved hypopituitarism & $30 \%$ & $20 \%$ & 0.653 \\
\hline Non-NE signs \& symptoms & & & \\
\hline$\quad$ Improved headache & $85 \%$ & $79 \%$ & 1.0 \\
\hline$\quad$ Improved encephalopathy & $100 \%$ & $100 \%$ & - \\
\hline$\quad$ Improved nuchal rigidity & $100 \%$ & $100 \%$ & - \\
\hline$\quad$ Improved nausea/vomiting & $100 \%$ & $100 \%$ & - \\
\hline - = not applicable. & & &
\end{tabular}

nausea and vomiting $(\mathrm{p}=1.0$, Fisher's exact test) between the early and delayed treatment patients. These symptoms tended to improve after surgery (Table 4), and patients undergoing early surgery were no more likely than those undergoing delayed surgery to show improvement in these nonneuroendocrine signs and symptoms: 10 (77\%) of 13 patients in the early treatment group showed improvement versus $15(83 \%)$ of 18 patients in the delayed treatment group ( $\mathrm{p}=0.676$, Fisher's exact test).

\section{Outcomes Based on Varied Time to Surgery From Symptom Onset and Time of Admission}

Overall, 2 patients $(6 \%)$ underwent surgery within 24 hours of symptom onset, 5 (16\%) at 48 hours from symptom onset, and $6(19 \%)$ at 72 hours from symptom onset. Additional analysis revealed that separating patients based on a 48 -hour time point rather than a 72 -hour time point was no more likely to show recovery of neurological, endocrine, or nonneuroendocrine function than those treated beyond 48 hours.

When considering time to surgery from symptom onset as a continuous variable, there was no significant association between improved neurological, endocrine, or nonneuroendocrine function and time until surgery. This also held true when considering time to surgery from hospital admission: there was no association between time to the operating room from admission and improved neurological, endocrine, or nonneuroendocrine function.

\section{Discussion}

Given the complex presentations and outcomes associated with pituitary apoplexy, we sought to better analyze the importance of surgical timing in the treatment of acute pituitary apoplexy. We focused solely on surgical treatment rather than medical therapy, as our patient cohort overwhelmingly presented with evidence of visual deficits and/or cranial neuropathies and all underwent resection as a result.
In contrast to "subacute" or "subclinical" apoplexy in which radiographic evidence of tumor hemorrhage or infarction is seen with atypical or absent clinical symptoms, "acute" apoplexy was the exclusive focus of our series. Two recent studies comparing subclinical and classic apoplexy determined that subclinical cases occur more frequently than the classic and show comparable visual and endocrine recovery, ${ }^{17,37}$ but we believe that subclinical cases represent a different pathological process and did not include similar cases in our cohort.

Our male preponderance of patients reflects a slight gender gap seen in other larger studies of apoplexy, $4,18,26$ in which men appear to outnumber women by $2: 1$. Similarly, our mean age of 49 years reflects the more common distribution of patients in their 5th and 6th decades of life at presentation. ${ }^{30}$ All tumors in our case series were macroadenomas, and as occurs in the published literature, $3,4,18,23,26,27$ our case series comprised mostly nonfunctional adenomas (70\%), with only $15 \%$ of adenomas demonstrating hormone hypersecretion (3 prolactinomas, 1 somatotrophinoma). The fact that apoplexy is more often associated with larger tumors, which is well reported in the literature, $6,7,18,20,23,30,33,36$ is purportedly due to the increased tumor size outstripping blood supply and resulting in ischemia and/or hemorrhage. It has been suggested that features intrinsic to the biology of apoplectic adenomas, such as high metabolic demand with concomitant limited vascular supply, hypoglycemia, and limited nutrient supply, may predispose to ischemia and hemorrhage. ${ }^{24}$

Despite an increasingly nuanced view of treatment for acute pituitary apoplexy in which the literature has revealed an expanding role for nonsurgical conservative therapy in select cases - most often those cases without vi-

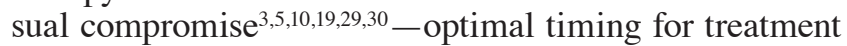
remains unclear. This combined with anecdotal evidence that apoplectic tumors may regress following conservative management ${ }^{12,38}$ makes it increasingly evident that acute pituitary apoplexy is not a uniform diagnosis or disease entity. Our longstanding practice has been emergent resection for acute pituitary apoplexy following preoperative endocrinological and medical stabilization, for example, normalization of hypocortisolism, hypothyroidism, and hyponatremia. This practice most likely reflects the high rate of visual compromise in our cohort; we report that 31 (97\%) of 32 patients possessed some form of visual compromise in the form of visual loss and/or CN III, IV, and/ or VI neuropathies, which prompted our group to perform endonasal transsphenoidal resection to remove tumor and relieve mass effect. Thus, while our study is not equipped for an analysis of medical versus surgical outcome, our similar rates of baseline neurological and nonneurological deficits allowed us to perform a head-to-head comparison among patients undergoing early versus delayed surgical treatment. Delays in time to treatment were attributable to delayed patient presentation to medical attention or to transfer delays from referring institutions.

The only differences in baseline characteristics between our 2 patient cohorts were the higher prevalence of hypopituitarism seen in the delayed treatment cohort and higher rates of GTR in the early treatment group. We interpret the former finding as most likely reflective of the 
delayed manner in which ischemia and infarction of an apoplectic adenoma cause glandular compromise and necrosis, whereas the latter finding suggests that the loss of tumor architecture, pseudocapsule, and glandular interface in apoplectic tumors may make it more difficult to achieve GTR. Our analysis of hemorrhagic tumors and extent of resection showed no association between hemorrhage and resectability, raising the possibility that a tumor's gross consistency may not be the only factor predicting resectability and that features intrinsic to apoplectic tumors may render them more difficult to resect following an initial apoplectic infarction or hemorrhage.

While statistically insignificant, the trend toward greater visual loss and field cuts at presentation for patients who underwent early rather than delayed surgery is nevertheless an important caveat for our study. It raises the possibility that early treatment of more severe symptomatology may be necessary to ensure outcomes similar to patients undergoing delayed treatment of more moderate symptomatology and that our patient numbers were simply underpowered to detect a bias toward treating certain apoplexy patients earlier. Indeed, the literature frequently glosses over the use of surgical intervention for "more severe" cases versus conservative medical therapy for patients without acute vision loss or ophthalmoplegia, but this bias toward treating apoplectic patients differently based on acuity of presentation makes it impossible to draw firm conclusions about ideal treatment. We have shown a statistically insignificant $(p=0.07)$ difference in baseline rates of visual deficits between our 2 treatment groups, allowing us to more accurately determine the lack of an association between treatment timing and outcome, though more robust patient numbers collected in a prospective fashion would undoubtedly bolster our argument and strengthen our statistics.

A litany of retrospective studies ${ }^{1,3-5,10,15,18,19,26,29}$ have attempted to stratify patients by optimal medical versus surgical management but have yielded largely conflicting results; a recent meta-analysis suggested an improvement in visual and endocrine outcomes in patients undergoing resection ${ }^{31}$ but reviewed too few studies to claim statistical significance and included only retrospective studies. Few papers directly address the matter of optimal timing of surgical intervention independent of comparisons to medical therapy, and those that do have also yielded conflicting results.

In a 2016 study of 31 patients with classic pituitary apoplexy, Giritharan and colleagues ${ }^{8}$ treated 11 patients conservatively, 11 with emergent surgery within 7 days of presentation, and 9 with surgery more than 7 days from presentation. The authors did not find any statistically significant differences in the rates of visual or endocrine recovery among the groups, despite the fact that the emergent surgery cohort possessed higher rates of baseline visual and endocrine dysfunction. Bujawansa and colleagues ${ }^{5}$ similarly concluded that there was no clear visual, $\mathrm{CN}$, or endocrine benefit to early surgical ( $<7$ days) versus late surgical ( $>7$ days) intervention, either in a head-to-head comparison or when compared with medical management, with the major caveat that Pituitary Apoplexy Scores were significantly higher in the early surgery group. Singh et al. ${ }^{30}$ performed a similar study on medical versus early and delayed surgery for acute apoplexy, concluding that medical therapy was a viable treatment option given the lack of clear benefit seen among the surgical cohorts. Their study did not use a fixed cutoff for early versus delayed surgery, however, and patients undergoing early surgery tended to have more severe visual deficits at presentation.

In contrast, Bills et al. ${ }^{4}$ published the results of their surgical management of apoplexy prior to 1992 and affirmed a role for early surgery, defined as $<7$ days, with statistically significant improvements seen among patients with visual deficits. Interestingly, a subgroup analysis of patients who underwent surgery within 3 days of presentation versus 4-7 days did not show a difference in visual outcomes, similar to findings in our study. Randeva and colleagues ${ }^{26}$ similarly concluded that patients undergoing resection within 8 days of symptom onset demonstrated significantly superior visual outcomes compared with those undergoing delayed surgery, though their study did not find a difference in rates of ocular paresis improvement. Seuk et al. ${ }^{28}$ showed a statistically significant improvement in visual acuity and field cuts among patients undergoing resection within 48 hours of symptom onset, while Woo et al. showed a similar improvement in visual deficits with early surgery $(<3$ days) versus delayed surgery. ${ }^{35}$

Endocrine recovery appears to be poor regardless of timing of intervention, consistent with our own findings. While one study ${ }^{2}$ demonstrated improved endocrinological outcomes following early surgery, the case series involved only 8 patients, and numerous other groups have reported uniformly poor endocrine outcomes. . $, 8,10,18,29,30^{2}$ We found no difference in the rates of hormone recovery between the early and delayed groups. Importantly, we only included patients who had undergone postoperative endocrine testing at least 6 months postoperatively to allow enough time for possible reestablishment of the hypothalamic-pituitary axis. The limited number of patients demonstrating endocrine recovery likely represents those with a stunned but intact pituitary gland. Patients should be counseled that the overall likelihood of the return of function is low and that delayed recovery is unusual.

Nonneuroendocrine symptoms improved regardless of the timing of treatment. Many of the symptoms, such as headache and nuchal rigidity, can be caused by mass effect and are likely to improve with time, regardless of the onset of intervention, whereas systemic features such as nausea/vomiting and encephalopathy can be exacerbated by underlying endocrinopathy and thus are best treated medically.

Importantly, when resection is performed by experienced pituitary surgeons, patients can be counseled that neurological outcomes following surgery remain favorable for acute pituitary apoplexy, despite poor outcomes for the reversal of endocrinopathy. Given the breadth of patient age, presentation, tumor size, hormonal functionality, and variable time from presentation until surgery, we feel our results are externally valid and can apply to other patients who present with pituitary apoplexy.

Our findings suggest that delays in clinical presentation do not adversely impact outcomes in apoplexy patients. Our findings also suggest that time can be taken to pre- 
operatively correct endocrinopathies, obtain high-quality imaging for surgical planning, and allow patients to come under the care of experienced pituitary surgeons at dedicated pituitary centers of excellence ${ }^{21}$ without adversely affecting apoplexy patient outcomes. Nevertheless, we still advocate transsphenoidal surgery without excessive delay for apoplexy patients who present with visual deficits given the clear benefits to surgery, regardless of timing.

\section{Study Limitations}

As a retrospective case series, our study is inherently limited by selection bias and incomplete documentation or follow-up for all patients. The patients lost to follow-up limit our ability to perform more meaningful neurological and endocrinological postoperative analyses. Where possible, we attempted to limit bias by capturing all apoplexy patients regardless of outcome and to maximize follow-up intervals. While our analyses showed that defining early versus delayed surgery at 48 hours or 72 hours from symptom onset to resection resulted in similar neurological, endocrine, and nonneuroendocrine outcomes, our final selection of 72 hours was chosen to maximize statistical power. A greater number of patients in the earlier treatment groups ( 24 hours, 48 hours) would facilitate more meaningful statistical analysis.

\section{Conclusions}

Acute pituitary apoplexy presents with derangements of neurological and endocrinological function. Neurological deficits such as visual loss and cranial neuropathies show improvement following surgical decompression, with rarer recovery from hypopituitarism, and most patients require long-term hormone replacement. The timing of surgical intervention relative to the onset of symptoms does not appear to significantly affect the resolution of neurological or endocrinological deficits.

\section{Acknowledgments}

Dr. Rutkowski received a Precision Health Science TL1 (PRESCIENT) Postdoctoral Fellowship training grant.

\section{References}

1. Allen AM, Pawlicki T, Dong L, Fourkal E, Buyyounouski M, Cengel K, et al: An evidence based review of proton beam therapy: the report of ASTRO's emerging technology committee. Radiother Oncol 103:8-11, 2012

2. Arafah BM, Harrington JF, Madhoun ZT, Selman WR: Improvement of pituitary function after surgical decompression for pituitary tumor apoplexy. J Clin Endocrinol Metab 71:323-328, 1990

3. Ayuk J, McGregor EJ, Mitchell RD, Gittoes NJL: Acute management of pituitary apoplexy-surgery or conservative management? Clin Endocrinol (Oxf) 61:747-752, 2004

4. Bills DC, Meyer FB, Laws ER Jr, Davis DH, Ebersold MJ, Scheithauer BW, et al: A retrospective analysis of pituitary apoplexy. Neurosurgery 33:602-609, 1993

5. Bujawansa S, Thondam SK, Steele C, Cuthbertson DJ, Gilkes $\mathrm{CE}$, Noonan C, et al: Presentation, management and outcomes in acute pituitary apoplexy: a large single-centre experience from the United Kingdom. Clin Endocrinol (Oxf) 80:419-424, 2014
6. da Motta LA, de Mello PA, de Lacerda CM, Neto AP, da Motta LD, Filho MF: Pituitary apoplexy. Clinical course, endocrine evaluations and treatment analysis. J Neurosurg Sci 43:25-36, 1999

7. Giammattei L, Mantovani G, Carrabba G, Ferrero S, Di Cristofori A, Verrua E, et al: Pituitary apoplexy: considerations on a single center experience and review of the literature. $\mathbf{J}$ Endocrinol Invest 39:739-746, 2016

8. Giritharan S, Gnanalingham K, Kearney T: Pituitary apoplexy-bespoke patient management allows good clinical outcome. Clin Endocrinol (Oxf) 85:415-422, 2016

9. Glick RP, Tiesi JA: Subacute pituitary apoplexy: clinical and magnetic resonance imaging characteristics. Neurosurgery 27:214-219, 1990

10. Gruber A, Clayton J, Kumar S, Robertson I, Howlett TA, Mansell P: Pituitary apoplexy: retrospective review of 30 patients - is surgical intervention always necessary? $\mathbf{B r} \mathbf{~ J}$ Neurosurg 20:379-385, 2006

11. Hage R, Eshraghi SR, Oyesiku NM, Ioachimescu AG, Newman NJ, Biousse V, et al: Third, fourth, and sixth cranial nerve palsies in pituitary apoplexy. World Neurosurg 94:447-452, 2016

12. Jackson DL, Van Gompel JJ: Rapid pituitary apoplexy regression: what is the time course of clot resolution? Case Rep Radiol 2015:268974, 2015

13. Jahangiri A, Clark AJ, Han SJ, Kunwar S, Blevins LS Jr, Aghi MK: Socioeconomic factors associated with pituitary apoplexy. J Neurosurg 119:1432-1436, 2013

14. Knosp E, Steiner E, Kitz K, Matula C: Pituitary adenomas with invasion of the cavernous sinus space: a magnetic resonance imaging classification compared with surgical findings. Neurosurgery 33:610-618, 1993

15. Leyer C, Castinetti F, Morange I, Gueydan M, Oliver C, Conte-Devolx B, et al: A conservative management is preferable in milder forms of pituitary tumor apoplexy. J Endocrinol Invest 34:502-509, 2011

16. Liu JK, Couldwell WT: Pituitary apoplexy in the magnetic resonance imaging era: clinical significance of sphenoid sinus mucosal thickening. J Neurosurg 104:892-898, 2006

17. Liu ZH, Chang CN, Pai PC, Wei KC, Jung SM, Chen NY, et al: Clinical features and surgical outcome of clinical and subclinical pituitary apoplexy. J Clin Neurosci 17:694-699, 2010

18. Lubina A, Olchovsky D, Berezin M, Ram Z, Hadani M, Shimon I: Management of pituitary apoplexy: clinical experience with 40 patients. Acta Neurochir (Wien) 147:151-157, 2005

19. Maccagnan P, Macedo CL, Kayath MJ, Nogueira RG, Abucham J: Conservative management of pituitary apoplexy: a prospective study. J Clin Endocrinol Metab 80:2190-2197, 1995

20. McFadzean RM, Doyle D, Rampling R, Teasdale E, Teasdale G: Pituitary apoplexy and its effect on vision. Neurosurgery 29:669-675, 1991

21. McLaughlin N, Laws ER, Oyesiku NM, Katznelson L, Kelly DF: Pituitary centers of excellence. Neurosurgery 71:916926, 2012

22. Milazzo S, Toussaint P, Proust F, Touzet G, Malthieu D: Ophthalmologic aspects of pituitary apoplexy. Eur J Ophthalmol 6:69-73, 1996

23. Möller-Goede DL, Brändle M, Landau K, Bernays RL, Schmid C: Pituitary apoplexy: re-evaluation of risk factors for bleeding into pituitary adenomas and impact on outcome. Eur J Endocrinol 164:37-43, 2011

24. Oldfield EH, Merrill MJ: Apoplexy of pituitary adenomas: the perfect storm. J Neurosurg 122:1444-1449, 2015

25. Onesti ST, Wisniewski T, Post KD: Clinical versus subclinical pituitary apoplexy: presentation, surgical management, and outcome in 21 patients. Neurosurgery 26:980-986, 1990 
26. Randeva HS, Schoebel J, Byrne J, Esiri M, Adams CBT, Wass JAH: Classical pituitary apoplexy: clinical features, management and outcome. Clin Endocrinol (Oxf) 51:181188,1999

27. Semple PL, Jane JA Jr, Laws ER Jr: Clinical relevance of precipitating factors in pituitary apoplexy. Neurosurgery 61:956-962, 2007

28. Seuk JW, Kim CH, Yang MS, Cheong JH, Kim JM: Visual outcome after transsphenoidal surgery in patients with pituitary apoplexy. J Korean Neurosurg Soc 49:339-344, 2011

29. Sibal L, Ball SG, Connolly V, James RA, Kane P, Kelly WF, et al: Pituitary apoplexy: a review of clinical presentation, management and outcome in 45 cases. Pituitary 7:157-163, 2004

30. Singh TD, Valizadeh N, Meyer FB, Atkinson JL, Erickson D, Rabinstein AA: Management and outcomes of pituitary apoplexy. J Neurosurg 122:1450-1457, 2015

31. Tu M, Lu Q, Zhu P, Zheng W: Surgical versus non-surgical treatment for pituitary apoplexy: a systematic review and meta-analysis. J Neurol Sci 370:258-262, 2016

32. Turgut M, Ozsunar Y, Başak S, Güney E, Kir E, Meteoğlu I: Pituitary apoplexy: an overview of 186 cases published during the last century. Acta Neurochir (Wien) 152:749-761, 2010

33. Verrees M, Arafah BM, Selman WR: Pituitary tumor apoplexy: characteristics, treatment, and outcomes. Neurosurg Focus 16(4):E6, 2004

34. Vidal E, Cevallos R, Vidal J, Ravon R, Moreau JJ, Rogues AM, et al: Twelve cases of pituitary apoplexy. Arch Intern Med 152:1893-1899, 1992

35. Woo HJ, Hwang JH, Hwang SK, Park YM: Clinical outcome of cranial neuropathy in patients with pituitary apoplexy. $\mathbf{J}$ Korean Neurosurg Soc 48:213-218, 2010
36. Zhan R, Li X, Li X: Endoscopic endonasal transsphenoidal approach for apoplectic pituitary tumor: surgical outcomes and complications in 45 patients. J Neurol Surg B Skull Base 77:54-60, 2016

37. Zhang F, Chen J, Lu Y, Ding X: Manifestation, management and outcome of subclinical pituitary adenoma apoplexy. $\mathbf{J}$ Clin Neurosci 16:1273-1275, 2009

38. Zieliński G, Witek P, Koziarski A, Podgórski J: Spontaneous regression of non-functioning pituitary adenoma due to pituitary apoplexy following anticoagulation treatment - a case report and review of the literature. Endokrynol Pol 64:54-58, 2013

\section{Disclosures}

The authors report no conflict of interest concerning the materials or methods used in this study or the findings specified in this paper.

\section{Author Contributions}

Conception and design: Rutkowski. Acquisition of data: Rutkowski. Analysis and interpretation of data: all authors. Drafting the article: Rutkowski. Critically revising the article: all authors. Reviewed submitted version of manuscript: all authors. Approved the final version of the manuscript on behalf of all authors: Rutkowski. Statistical analysis: Rutkowski. Study supervision: Kunwar, Blevins, Aghi.

\section{Correspondence}

Martin J. Rutkowski, Department of Neurological Surgery, University of California, San Francisco, 505 Parnassus Ave., M-779, San Francisco, CA, 94143. email: martin.rutkowski@ucsf.edu. 